\title{
Classification of ice crystals at C-band
}

\author{
J. Steinert and M. Chandra \\ Chair of Microwave Engineering and Photonics, Chemnitz University of Technology, Germany
}

\begin{abstract}
Clouds consist of water particles (hydrometeors) in different aggregate states. Above the melting layer these hydrometeors are formed mainly as ice crystals and other completely or partly frozen particles.

With measurements from the C-band dual polarimetric radar POLDIRAD (Oberpfaffenhofen, Germany) the backscattered signals of the ice particles in the horizontal/vertical polarisation base were analysed. The focus is lying on the co-polar reflectivities and therefore the differential reflectivity. In the next step a simulation of the backscattered signals deliver the physical ansatz for the creation of an ice crystal class. Finally the comparison of this class with a raindrop classification is shown.
\end{abstract}

\section{Introduction}

The analysis of ice crystals using C-band radar is a rarely addressed topic in the hydrometeor observation. Due to their small size (typically just tenths of millimetres) it is more useful to use higher observation frequencies in the range of $100 \mathrm{GHz}$ or higher with their smaller wavelengths in the area of millimetres (e.g. Wolde and Vali, 2001 and Aydin and Singh, 2002). Therefore the investigation with a cmwavelength radar lead to lower echo intensities as provided by a mm-wavelength radar. Furthermore, single ice particles have very high axial-ratio-shapes (Sect. 3) and with it the usage of dual polarimetric radar (Sect. 2) give additional information.

The paper starts with a short description of the radar hardware and measured datasets in Sect. 2 followed by the introduction of the model for the backscattering simulation of the ice particles in Sect. 3. The comparison of the measured

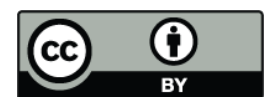

Correspondence to: J. Steinert

(joerg.steinert@etit.tu-chemnitz.de) powers with the simulated values is then given in Sect. 4 . The paper is finalized in Sect. 5 with the application of the achieved results in a classification algorithm.

\section{Hardware for measurement and measured signatures}

With the weather radar of the DLR (POLDIRAD, Sect. 2.1) datasets of different precipitation events (stratiform and convective) including ice particles in cirrus formations were investigated. From this database, regions of ice crystals were manually selected (Sect. 2.2) and from the resulting scatter plots of the reflectivity and the differential reflectivity a corresponding fitting function was determined (Sect. 2.3).

\subsection{POLDIRAD and the measured parameters}

The POLDIRAD is a ground-based dual polarisation radar and is located in Oberpfaffenhofen (near Munich), Germany. The co-polar (eg. $Z_{h h}$ and $Z_{v v}$ for a horizontal/vertical polarisation base) and cross-polar reflectivities $\left(Z_{h v}\right.$ and $\left.Z_{v h}\right)$ are achieved from several samples (typically 128). Each sample is measured within a time interval of two pulses (cf., the "Smatrix" mode measurement scheme in Fig. 1a from Schroth et al., 1988). The minimum detectable signal of $-108 \mathrm{dBm}$ (cf., Table 3 in Schroth et al., 1988) makes the recognition of ice crystal signatures possible with their very low backscattered signals. For the analysis of the data the two co-polar reflectivities $Z_{h h}$ and $Z_{v v}$ measured in $\mathrm{dBZ}$ were taken. The indices mean that the polarisation of the transmitted and the received signal is the same. Another parameter which follows is the differential reflectivity $Z_{D R}$ with

$Z_{D R}=Z_{h h}-Z_{v v}$

resulting in $\mathrm{dB}$. 


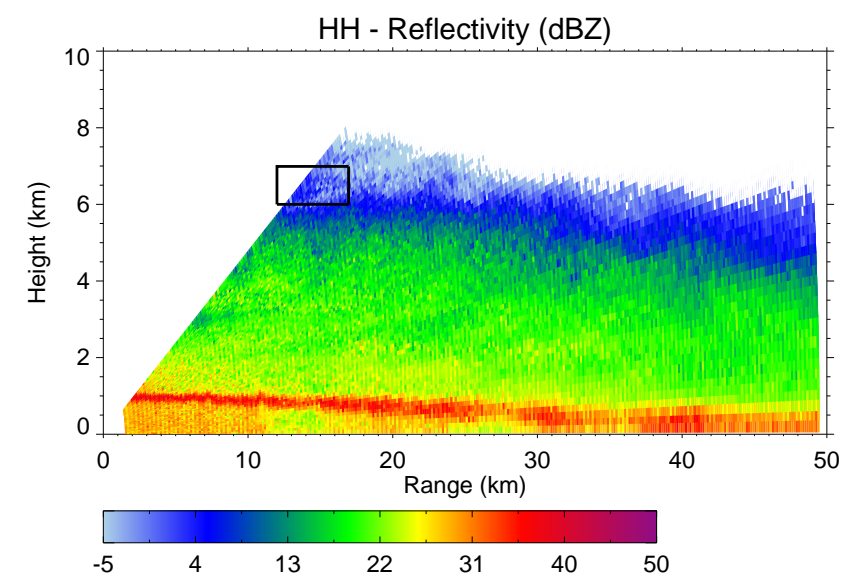

Fig. 1. Selection of ice crystal signatures (black rectangle) of an RHI plot of the co-polar reflectivity $Z_{h h}$ recorded on the $10^{\text {th }}$ of November 1994.

\subsection{Analysis of the datasets}

Ice crystal data has been selected from several datasets (independent from stratiform and convective clouds for different seasons). The selection of the ice crystal signatures has been done at the top layer of the clouds. The occurence in this region is resulted due to cloud dynamics and low temperatures above the $0^{\circ} \mathrm{C}$ isotherme. Important is the fact that the radar processor delivers the equivalent reflectivity $\left(Z_{e}\right)$. This means that the reflectivity is connected to scatterers in liquid phase. More details to the relation between the aggregate state and the backscattered signal are given in Sect. 3. For the present it is only meaningful that the reflectivities in the forthcoming plots and equations are with $Z_{e}$. One example of the data selection is shown in Figs. 1 and 2 with a black rectangle.

The ice crystal data of seven datasets is plotted in Fig. 3 as scatter plot of the horizontal reflectivity and the differential reflectivity. As can be seen in the scatter plot $Z_{D R}$ (a parameter that is closely related to the particle shape at C-band) reaches values up to $4 \mathrm{~dB}$ and higher. This corresponds to very oblate hydrometeors (cf., Steinert and Chandra, 2008). The reflectivity in horizontal polarisation reaches minimum values downto $-10 \mathrm{dBZ}$ which demonstrates the weakness of the backscattered signals from ice crystal signatures. It has to be mentioned that no comparable in-situ measurements were available to prove that there are ice crystals in the chosen cases. But generally in-situ measurements by aircrafts (e.g. Wolde and Vali, 2001) have shown that ice crystal structures exist in the top layer of clouds.

\subsection{Fitting function}

The summary of data to an equation is not easy to handle. Above all, a fitting function is a challenge for data with large

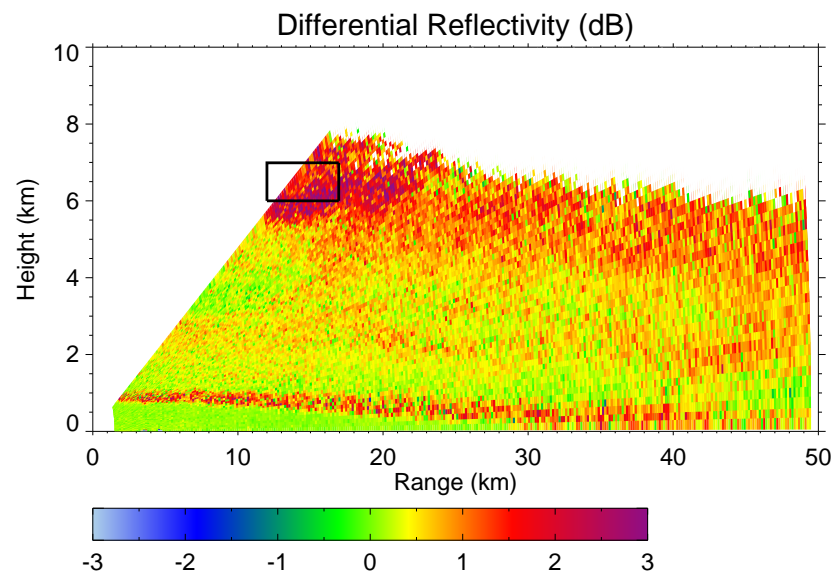

Fig. 2. RHI plot of the differential reflectivity $Z_{D R}$, the same dataset as in Fig. 1.

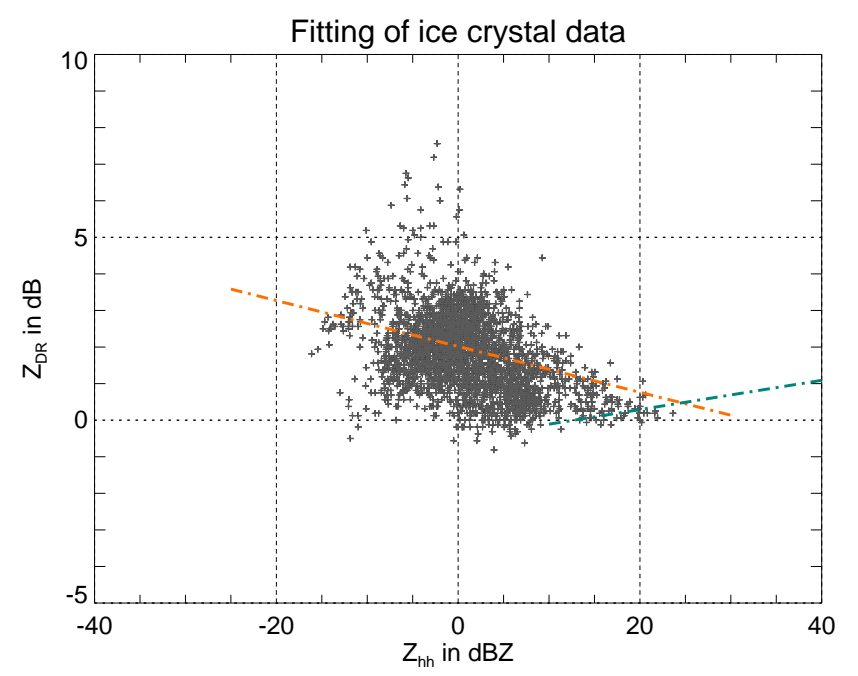

Fig. 3. Radar data (manually selected out of regions with ice crystals) of $Z_{h h}$ and $Z_{D R}$ (orange line is the linear fitting function of the ice crystals; green line is the fitting function of raindrop data Steinert and Chandra, 2008).

variations and so the chosen type of fitting function is the linchpin of the mathematical and optical accuracy. Here, a linear fit was taken though we have to keep in mind that other types (e.g. exponential) will fit the data, too. The equation for the fit is

$$
Z_{D R}=-0.063 Z_{h h}+2.02 \text {. }
$$

Shown is this function in orange color in Fig. 3 as overplot to the data. 


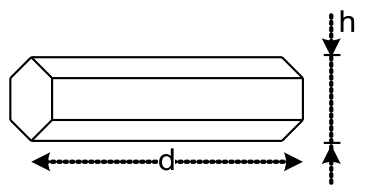

a)

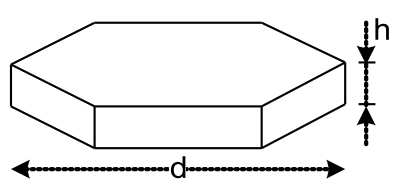

b)

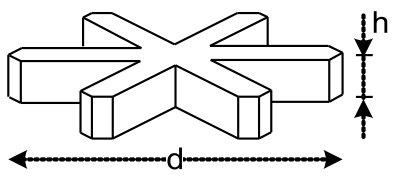

c)

Fig. 4. Models of the three types of shapes of ice crystals. (a) column, (b) plate, (c) stellar according to Auer and Veal (1970).

\section{Simulation of backscattered signals from ice crystals}

The reflectivity is calculated by

$\zeta_{h h, v v}=\frac{\lambda^{4}}{\pi^{5} \cdot|K|^{2}} \cdot \int_{D_{e}} \sigma_{h h, v v}\left(D_{e}\right) \cdot N\left(D_{e}\right) d D_{e}$

with the wavelength $\lambda$ of the transmitted/received signal, $K$ the dielectric factor calculated by $K=\frac{\epsilon-1}{\epsilon+2}$ and the complex permittivity $\epsilon . \sigma_{h h, v v}$ is the backscattering crosssection for the corresponding polarisation. Because of the high dynamic range of the reflectivity values from Eq. 3, the transformation into the logarithmic scale is obvious with $Z_{h h, v v}=10 \cdot \log _{10}\left(\zeta_{h h, v v}\right)$. The result is $Z_{h h, v v}$ in the unit $\mathrm{dBZ}$ (cf., Sect. 2.1). The differential reflectivity $Z_{D R}$ that is related to the shape of the hydrometeors is then estimated with Eq. 1. The permittivity was calculated according to Ray (1972) and results for ice crystals to $|K|^{2} \approx 0.176$ and for water to $|K|^{2} \approx 0.931$. This difference is important for the consideration of $Z_{e}$ because it follows for the comparison of the simulation with the data $\zeta_{e}=\zeta_{\text {water }}=\frac{\zeta_{\text {ice }}}{5.29}$ or $Z_{e}=Z_{\text {ice }}-7.23 \mathrm{~dB}$ (cf., Eq. 3).

Ice particles have various shapes which were characterised in the three types column, plate and stellar by Auer and Veal (1970). They are shown in Fig. 4 and the corresponding height $h$ to diameter $d$ relations from Auer and Veal (1970) are given by

columns: $h=11.3 d^{0.414}$

plates: $h=2.02 d^{0.449}$

stellars: $h=2.03 d^{0.431}$

with $h$ and $d$ in $\mu \mathrm{m}$.

$\sigma_{h h, v v}$ for ice particles are simulated with the RayleighGans approximation and so with the calculations for spheroids. This simplification in shape was done because the small size of ice particles compared to the wavelength leads to a small error by using a variant shape for plates and columns (Vivekanandan et al., 1994). The dropsize distribution (DSD) of the particles was asummed as exponential with $N\left(D_{e}\right)=3.31 \cdot 10^{5} \cdot e^{-3.67 \cdot \frac{D_{e}}{D_{0}}}\left[\frac{1}{\mathrm{~mm} \cdot \mathrm{m}^{3}}\right]$ (cf., Aydin and Walsh, 1998).

The simulated reflectivities are displayed with solid lines in Fig. 5 for the three types of ice crystals. Depending on the size relations and so the extent of oblateness of the particles (shown with Eq. 4) the $Z_{D R}$ is higher for plates and stellars as for columns. In Aydin and Walsh (1998) equations from simulation results were presented as $Z_{D P}{ }^{*}-Z_{h h}$ relationships. They were transformed to $Z_{D R}-Z_{h h}$ relationships and overplotted in Fig. 5 with dashed lines.

The mean squared error (MSE) with

$$
\operatorname{MSE}=\left(\operatorname{bias}\left(f_{0}(x)-f_{1}(x)\right)\right)^{2}+\operatorname{var}\left(f_{0}(x)-f_{1}(x)\right)
$$

was selected as criterium for the goodness-of-fit between two functions. Lower the MSE the better is the conformance of the two functions. It is to be seen in Fig. 5 that the functions for stellars give good results with $\operatorname{MSE}\left(Z_{D R}\right)_{s}=0.31$. The performance for plates and columns is weaker with $\operatorname{MSE}\left(Z_{D R}\right)_{p}=2.75$ for plates and $\operatorname{MSE}\left(Z_{D R}\right)_{c}=2.03$ for columns. An explanation for the errors can be linked to the different scattering models (e.g. the usage of a distribution for canting angle or DSD parameters in Aydin and Walsh, 1998). Depending on the fact that columns are not rotationally symmetric in their fall behaviour the backscattering cross-section changes with time. A point of criticism is then the, for this study implemented, view on a fixed position of the column with an averaged cross-section without taking into account the statistical distribution of the position.

\section{Combination of measurements with simulation re- sults}

In the following no mixing of the three types of ice particles was assumed and so only homogeneous volumes are presented. The differences between the results from the real measurement (Fig. 3) and the simulation (Fig. 5) show a high offset in $Z_{D R}$ values of $\operatorname{bias}\left(Z_{D R}\right)_{p}=7.2 \mathrm{~dB}$ for plates and $\operatorname{bias}\left(Z_{D R}\right)_{s}=7.3 \mathrm{~dB}$ for stellars. The columns are more similar with the data with $\operatorname{bias}\left(Z_{D R}\right)_{c}=2.6 \mathrm{~dB}$. To give better agreement to the data we focus on the variation of two parameter in the microphysical simulation. On the one hand, the canting of the particles represented by the canting angle $\alpha$ and on the other hand the permittivity $\epsilon$ of the particles was addressed. In the first stage the ice particles were implemented with horizontal orientation (no canting) (cf., Fig. 5). Then the canting angle was introduced by a method of Holt (1984). The best solution for columns was found

\footnotetext{
${ }^{*} Z_{D P}=10 \cdot \log _{10}\left(\zeta_{h h}-\zeta v v\right)$
} 


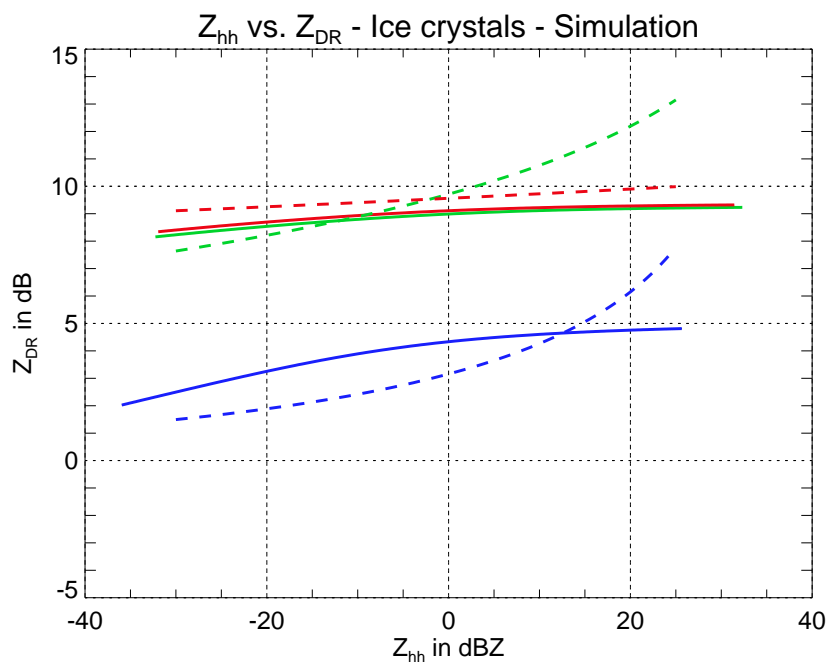

Fig. 5. $Z_{h h}-Z_{D R}$ plane of simulation results (solid lines) and corresponding functions from Aydin and Walsh (1998) (dashed lines) for the three ice crystal types (blue - columns; green - plates; red stellars).

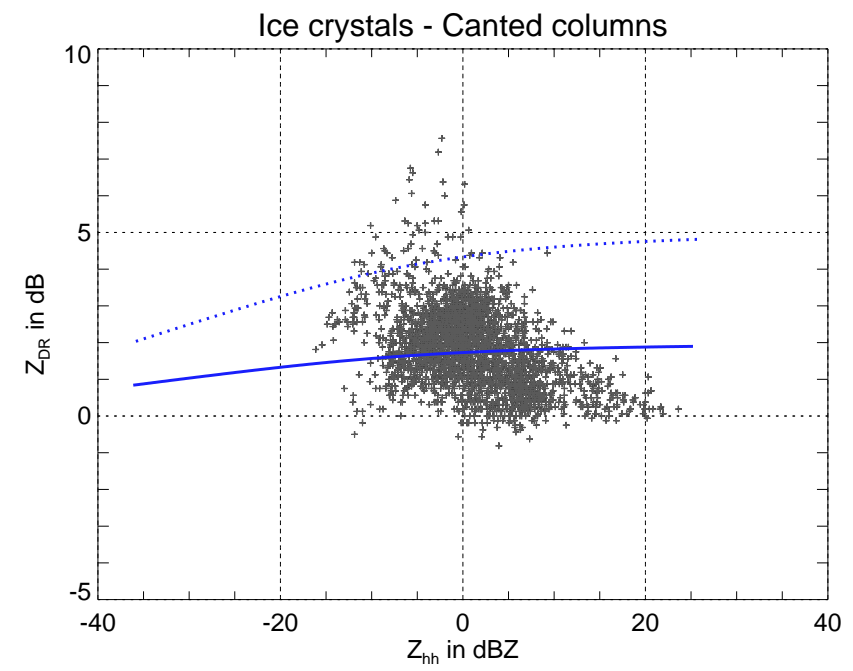

Fig. 6. $Z_{h h}-Z_{D R}$ plane of the selected ice crystal data with the simulation results of columns (solid blue curve $-\alpha=25^{\circ}$; dotted blue curve $-\alpha=0^{\circ}$ ).

with $\alpha=25^{\circ}$. The MSE reduces from $\operatorname{MSE}\left(Z_{D R}\right)_{c, 0^{\circ}}=8.06$ to $\operatorname{MSE}\left(Z_{D R}\right)_{c, 25^{\circ}}=1.21$ which is a good value if we look at the variance of the data. In Fig. 6 the simulation of the canted columns (blue solid curve) is displayed with the ice crystal data (dark grey color).

For the high decrease of the $Z_{D R}$ from plates and stellars the canting assumption alone does not match the data and so the second parameter the permittivity joined the game. The variation in $\epsilon$ means that the material of the scatterer

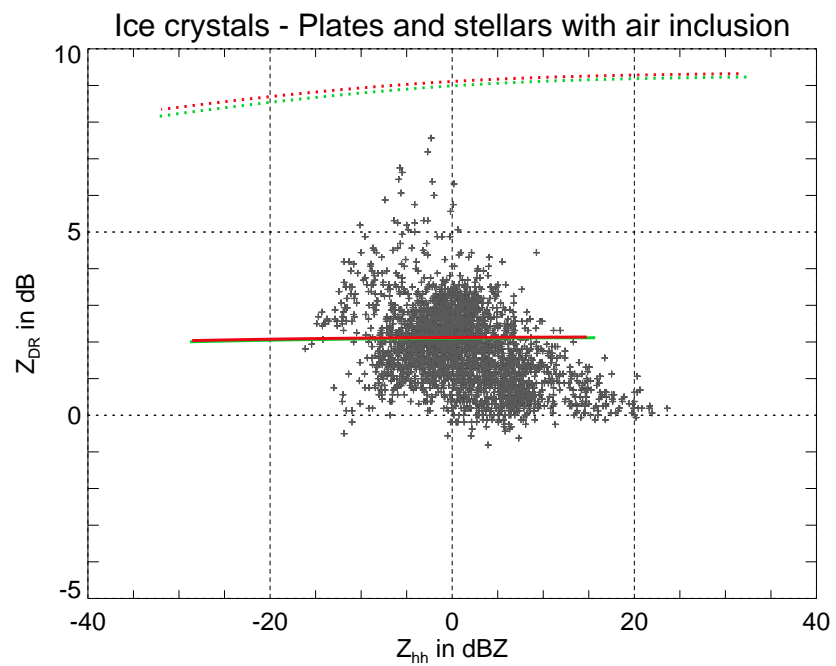

Fig. 7. $Z_{h h}-Z_{D R}$ plane of simulation results of plates (green) and stellars (red) with a density of $\rho_{\text {mix }}=0.2 \frac{\mathrm{g}}{\mathrm{cm}^{3}}$ (solid curves) and $\rho_{\text {ice }}$ (dotted curves) with the selected ice crystal data.

changes. In this case, the combination of pure ice with air inclusion was chosen, which represents the transfer from pure ice crystals to snowflakes. The mixing was done by

$$
\left(\frac{n_{m}^{2}-1}{n_{m}^{2}+2}\right) \frac{1}{\rho_{m}}=\left(\frac{n_{i}^{2}-1}{n_{i}^{2}+2}\right) \frac{1}{\rho_{i}}+\left(\frac{n_{a}^{2}-1}{n_{a}^{2}+2}\right) \frac{1}{\rho_{a}}
$$

with the density $\rho$ in $\frac{g}{\mathrm{~cm}^{3}}$ and the refractivity index $n$ of pure ice $(i)$, air $(a)$ and the mixture $(m)$. The constant values in this equation are $\rho_{i}=0.92 \frac{\mathrm{g}}{\mathrm{cm}^{3}}, \rho_{a}=1.341 \cdot 10^{-3} \frac{\mathrm{g}}{\mathrm{cm}^{3}}$ as well as $n_{i}=1.780$ and $n_{a}=1$ at a temperature of $-10^{\circ} \mathrm{C}$. The mixture density of $\rho_{m}=0.2 \frac{\mathrm{g}}{\mathrm{cm}^{3}}$ gives on the one hand passable values of MSE $E_{\text {Plates }}=1.23$ and MSE $E_{\text {Stellars }}=1.24$ and is on the other hand in good agreement with the estimation of Dissanayake et al. (1983) with $0.15 \frac{\mathrm{g}}{\mathrm{cm}^{3}}<\rho_{m}<0.45 \frac{\mathrm{g}}{\mathrm{cm}^{3}}$.

Shown are the new simulation curves for plates and stellars with solid curves in Fig. 7 in green (plates) and red (stellars) color. This assumed density for plates and stellars and likewise the canted columns show that their existence is possible with respect to the real radar data.

\section{Classification of frozen particles and raindrops}

Ice crystals and raindrops have, apart from their different aggregate state, several geometrical shapes. With this the backscattered signals from them shall show distinguishable signatures. The polarimetric signatures and therewith the classification features for raindrops have been used from Steinert and Chandra (2008). The main criterium is $Z_{h h}$ as it is usually lower for ice crystals than for raindrops resulting from their physical size and particle size distribution. An overlapping area is given at $Z_{h h}=20 \mathrm{dBZ} \pm 5 \mathrm{~dB}$ (see green 


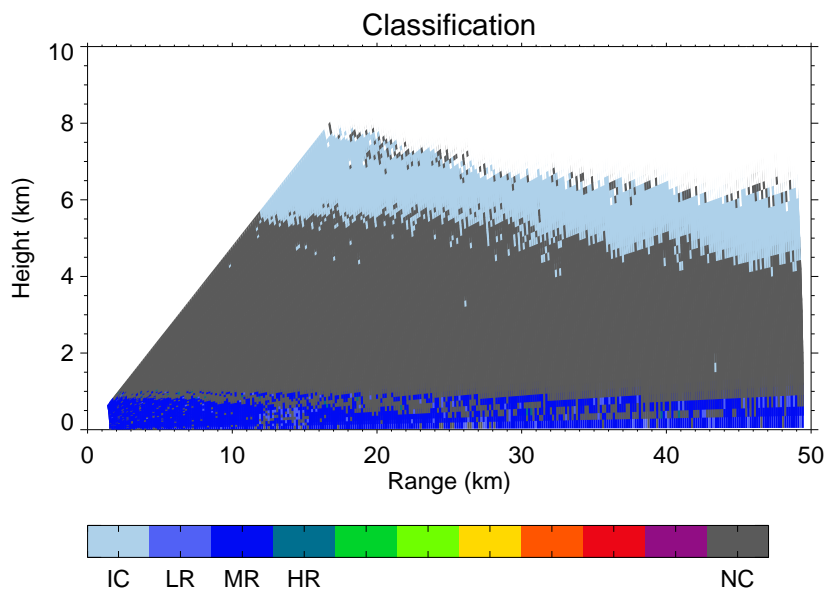

Fig. 8. Classification of the stratiform event of Fig. 1 (IC..ice crystals, LR..light rain, MR..medium rain, HR..heavy rain, NC..not classified).

and orange curve in Fig. 3) therefore a seperation between the two classes is just possible with an additional parameter. In the implemented classification the temperature, which was interpolated from the temperature at ground, was used. The classes for the raindrops were built from the equations of Steinert and Chandra (2008) and the fitting function of ice crystals (Eq. 2) is the base for the desired class. In Fig. 8 the classification of the same dataset as presented in Fig. 1 and $2\left(Z_{h h}\right.$ and $\left.Z_{D R}\right)$ is displayed. To increase the clarity the other hydrometeor classes like melting particles and snowflakes have been faded out from the picture and taken into the not classified (NC) class. This example shows that the seperation between ice crystals and raindrops succeeded well.

\section{Conclusions}

Starting with recorded C-band radar data of ice crystal signatures a comparison to simulation results was shown. The result is that for monodisperse volumes (only one type of plate, stellar or column) of ice crystal types, the simulation shall be expanded by a canting model. Additionally, the look on iceair mixed-phase particles is a promising way. The value of the mixed-phase permittivity for plates and stellars was in good agreement with Dissanayake et al. (1983) and the assumption of slightly canted columns delivers reasonable results. Furthermore, the formation of an ice crystal class gives with reflectivity and temperature parameters a well distinguishable classification between ice crystals and raindrops. Still a final statement of the hydrometeor composition in the radar pulse volume can not be given. But, relating to the subsun effect, as reflection of the sunlight by clouds (e.g. Katz, 1998), the suggestion of flat plates is prefered by the authors. For further studies, on the one hand, the combination of different ice crystal types and on the other hand the mixing of different frozen hydrometeor types like ice crystals with snowflakes could give better matching simulation results.

\section{References}

Auer, A. H. and Veal, D. L.: The dimensions of ice crystals in natural clouds, J. Atmos. Sci., 27, 919-926, 1970.

Aydin, K. and Singh, J.: Identification of cloud ice crystals using a $95 \mathrm{GHz}$ polarimetric radar, in: Geoscience and Remote Sensing Symposium Proceedings, 2002, IGARSS '02. 2002, IEEE Int., 5, 2814-2816, 2002.

Aydin, K. and Walsh, T.: Separation of millimeter-wave radar reflectivities of aggregates and pristine ice crystals in a cloud, in: Geoscience and Remote Sensing Symposium Proceedings, 1998, IGARSS '98, 1998 IEEE Int., 1, 440-442, 1998.

Dissanayake, A. W., Chandra, M., and Watson, P. A.: Prediction of differential reflectivity due to various types of ice particles and ice-water mixtures, in: ICAP 83, Norwich, UK, 56-59, IEE Publication No. 219, 1983.

Holt, A. R.: Some factors affecting the remote sensing of rain by polarization diversity radar in the 3 - to $35-\mathrm{GHz}$ frequency range, Radio Science, 19, 1399-1412, 1984.

Katz, J. I.: Subsuns and Low Reynolds Number Flow, J. Atmos. Sci., 55, 3358-3362, 1998.

Ray, P. S.: Broadband complex refractive indices of ice and water, Applied Optics, 11, 1836-1844, 1972.

Schroth, A. C., Chandra, M. S., and Meischner, P. F.: A C-Band Coherent Polarimetric Radar for Propagation and Cloud Physics Research, J. Atmos. Ocean. Techol., 5, 803-822, 1988.

Steinert, J. and Chandra, M.: Cloud physical properties and empirical polarimetric measurements of rain signatures at C-band, URSI Germany, Adv. Radio Sci., 6, 315-318, 2008, http://www.adv-radio-sci.net/6/315/2008/.

Vivekanandan, J., Bringi, V., Hagen, M., and Meischner, P.: Polarimetric radar studies of atmospheric ice particles, Geoscience and Remote Sensing, IEEE T., 32, 1-10, 1994.

Wolde, M. and Vali, G.: Polarimetric Signatures from Ice Crystals Observed at $95 \mathrm{GHz}$ in Winter Clouds. Part I: Dependence on Crystal Form, J. Atmos. Sci., 58, 828-841, 2001. 